\title{
The complex liaison between cachexia and tumor burden (Review)
}

\author{
ANDREA DE LERMA BARBARO \\ Biomedical Research Division, Department of Theoretical and Applied Sciences, \\ University of Insubria, Busto Arsizio, Varese, Italy
}

Received April 30, 2015; Accepted June 22, 2015

DOI: $10.3892 /$ or.2015.4164

\begin{abstract}
Cachexia is a wasting syndrome that afflicts end-stage cancer patients. Whereas a consensus statement for a definition of cachexia recently has been accomplished, a useful measurement for this condition at present is lacking. The aim of the present review is to discuss the advantage of introducing the measurement of tumor burden for a better overall evaluation of cachexia. Our suggestion ensues from a somewhat novel perspective in the field of infectious disease research where a careful measurement of the pathogen load, between i.e. different host genotypes, leads to the definition of the concept of tolerance to the infectious insult. Indeed tolerance concurs, together the more classical resistance, in maintaining the host reproductive fitness or health state. Noticeably a similar reasoning may apply to tumor biology as well. Whereas the extent of cachexia increases with tumor burden, the relationship between these two correlates of tumor progression fluctuates in a broad range. We have selected from the literature studies in the rodent model where significant variation in the course of the wasting illness during cancer was observed and quantitatively assessed comparing experimental groups marked by different genotype, drug treatment, diet or gender. These studies may be further classified in two categories: the former where the experimental condition associated to milder cachexia is accompanied to a lesser tumor burden, the latter where the inhibition of cachexia results disentangled from the tumor burden, that is the whole number of cancer cells results unchanged or even, paradoxically, is increased. In addition we survey, even in the context of human malignancy, the significance and feasibility of plotting quantitative estimates of cachexia against the whole tumor burden. Ultimately, the principal endeavor of introducing the measurement of tumor burden, in both experimental and clinical oncology, may be to achieve a better assessment of the inter-individual variation in the host vulnerability to cancer cachexia.
\end{abstract}

Correspondence to: Dr Andrea De Lerma Barbaro, Biomedical Research Division, Department of Theoretical and Applied Sciences, University of Insubria, via Alberto da Giussano 10, 21052 Busto Arsizio, Varese, Italy

E-mail: a.delermabarbaro@uninsubria.it; delermaa@libero.it

Key words: cachexia, muscle wasting, tumor burden, tolerance to infections, host vulnerability to cancer

\section{Contents}

1. Introduction

2. Mechanisms of cancer cachexia, a brief overview

3. A lesson from the field of infectious disease research, the utility of a measurement of tumor burden in the study of cachexia

4. Experimental oncology, rodent models

5. Cancer in humans: biased, hypothesis driven and unbiased approaches to the study of cachexia

6. Discussion

\section{Introduction}

Cachexia is a highly debilitating condition associated with poor physical function and quality of life (1-3). This wasting syndrome occurs in several life-threatening diseases such as cancer, congestive heart failure, rheumatoid arthritis, cystic fibrosis and Crohn's disease and in severe infectious diseases including AIDS and tuberculosis. In all these cases cachexia is a marker of poor prognosis and hence of end-stage disease. Conversely, the wasting syndrome observed in elderly people in the absence of obvious pathology is a somewhat separate noxious state most properly referred to as sarcopenia (4).

Cancer cachexia is characterized by pronounced weight loss and skeletal muscle atrophy. In fact cancer cachexia has recently been defined as 'a multifactorial syndrome characterized by an ongoing loss of skeletal muscle mass, with or without loss of fat mass, that cannot be fully reversed by conventional nutritional support and leads to progressive functional impairment' (5). The agreed diagnostic criterion for cachexia is unintentional weight loss $>5 \%$ (5), but the relevant physiological changes may be present long before this cutoff point is reached.

Many additional changes in normal heath state and physiology may be observed in cancer cachexia but not unfailingly; an incomplete list is anorexia, extreme fatigue, anemia, insulin resistance, edema and hypogonadism. In addition, the inflammatory response is widely regarded as the main driving force behind the metabolic alterations leading to cachexia $(3,6)$. However, cachexia can be present in the absence of overt systemic inflammation (5).

More than half of cancer patients suffer from cachexia and a substantial proportion of cancer patients die with symptoms of advanced cachexia. In fact, $>20 \%$ of cancer-related mortalities are thought to derive from cachexia, rather than 
from tumor burden and metastasis spreading. Even so, whether this wasting state is the ultimate cause of mortality in some patients is still unclear. In support of a direct cause of demise, cachexia may produce loss of respiratory muscle function leading to death from hypostatic pneumonia (7) or may cause myocardium atrophy leading to heart failure (8).

The greatest incidence of weight loss is observed among patients with some solid tumors, prominently gastric, pancreatic, lung, colorectal, as well as head and neck cancers. On the contrary, patients with breast cancer, sarcomas and hematological malignancies display the lowest frequency of weight loss combined with cachexia $(1,2)$.

Importantly weight loss in cancer patients is due to depletion of both adipose tissue and skeletal muscle mass, thus distinguishing cachexia from simple starvation. Moreover, weight loss in cancer patients is not merely the outcome of reduced calorie intake $(1,2)$.

Actually the metabolic changes found in cachexia resemble those of severe infection or injury rather than starvation and are multifactorial and complex. In particular, a frequent, relevant correlate of cachexia is a higher resting energy expenditure REE than in healthy controls; in addition, the protein catabolism leading to net body nitrogen loss concerns the muscle tissues, while visceral protein content is usually conserved and may even increase. This is the case in liver that in some cancer enlarges $(1,2)$.

The relationship between cancer therapy and cachexia is complex. Whereas the debulking of tumor mass by surgery or pharmacological treatment often leads to improvement in the cachectic state, chemotherapy and radiotherapy may induce anorexia and further weight loss. Actually the mechanism by which this worsening of health conditions occurs is believed to be different from that found in cancer cachexia (1), but this view is somewhat challenged by a direct wasting effect of cisplatin on skeletal muscle $(9,10)$.

Finally, not only cachexia predicts the overall survival rate, but it also indicates a trend towards lower chemotherapy response rates (11).

\section{Mechanisms of cancer cachexia, a brief overview}

As a preliminary remark, in the following discussion the term 'tumor' indicates the whole neoplastic tissue, including cancer cells and the mesenchymal stroma, such as the tumor microenvironment. Moreover, the rest of the host organism, the periphery, comprises bystander body compartments, variously involved in the neoplastic process and lastly, the target tissues of the wasting process (Fig. 1).

As a matter of fact cancer cachexia involves multiple tissues and pathways, producing a broad array of pathologic sequels. In hope of developing novel therapeutics to counteract this wasting state, current research is aimed at identifying the elusive mediators that cause i) loss of muscle tissue, ii) loss of adipose tissue, iii) high REE, iv) insulin resistance and metabolic imbalance, v) liver enlargement and acute phase response, vi) anorexia, vii) edema and finally, viii) hypogonadism.

In general, the mediators of cancer cachexia are thought to derive from i) the tumor, cancer cells or the inflammatory stroma, ii) bystander tissues/organs and iii) the targeted mesenchymal tissues undergoing wasting. Actually, a classi- fication based on the cell/tissue of origin may be misleading, since most cachectic factors can be produced by tumor as well as host tissues.

Many cachectic factors are broadly related to inflammation. Even though it is not included in the current clinical definition of cancer cachexia (5), systemic, smoldering inflammation is widely regarded as the underlying driver of the cancer-induced wasting process $(3,6)$. This chronic inflammatory state is triggered by the tumor itself, but is further sustained and amplified by bystander tissues/organs such as hypothalamus, adrenal gland or liver (Fig. 1).

An incomplete list of bona-fide cachectic factors encompasses the classical triad of inflammatory cytokines - TNF $\alpha$, IL1 $\beta$, IL6 and further includes the TH1 cytokine $\operatorname{IFN} \gamma(1-3,12)$. In particular $\mathrm{TNF} \alpha$, formerly named cachectin, facilitates skeletal muscle catabolism and contributes to insulin resistance, in part by impinging on the insulin signaling pathway (13). Moreover, IL6 is a major inducer of liver acute phase response (14).

Another putative cachectic factor connected with the resolution phase of the inflammatory process, but more broadly a key component of chronic stress response, is cortisol (15). Advanced cancer is associated with an array of symptoms, including pain, which can lead to activation of the neuroendocrine stress response. At large doses glucocorticoids facilitate myopathy in cancer cachexia and other wasting processes whereas at low doses these steroid hormones may produce the opposite outcome via inhibition of inflammatory cytokines.

While the aforementioned molecules are widely investigated in pathology, two cachexia-mediating factors studied almost exclusively in cancer biology in relation to cachexia are zinc- $\alpha 2$-glycoprotein ZAG, otherwise known as lipid mobilizing factor LMF, and proteolysis inducing factor PIF (1-3). Both factors possess selective activities capable of degrading adipose and skeletal muscle, respectively. Nowadays LMF/ ZAG and PIF are regarded as major candidates for the role of cancer cachexia promoters in humans. LMF/ZAG is produced by tumor as well as by normal tissues, such as liver and adipose tissue. Conversely, PIF production appears to be almost completely tumor-specific. It has been suggested that PIF is important during embryonic development and that in the adulthood only certain tumors may recover the ability to produce this molecule (16). However, more recently this conclusion has been challenged (17).

Finally, myostatin and other TGF $\beta$ family members, such as activin A, have captured the attention of the investigators involved in cachexia research. Actually, these probable cachectic factors are physiologic inducers of muscle hypotrophy and are referred to as TGF $\beta$-like myokines (18).

Of note, animals and humans that are genetically null for myostatin demonstrate dramatic muscle hypertrophy; conversely, experimentally forced myostatin overexpression results in a bias toward atrophy (19).

In normal physiology, myostatin and activins are synthesized and secreted mainly by skeletal muscle cells and used by these cells in an autocrine loop. There is growing evidence that the activity of the myostatin/activin pathway is upregulated in most catabolic diseases, thus contributing to the development of muscle wasting. Elevated serum activin A has been reported in various malignant diseases in mice as well as in 


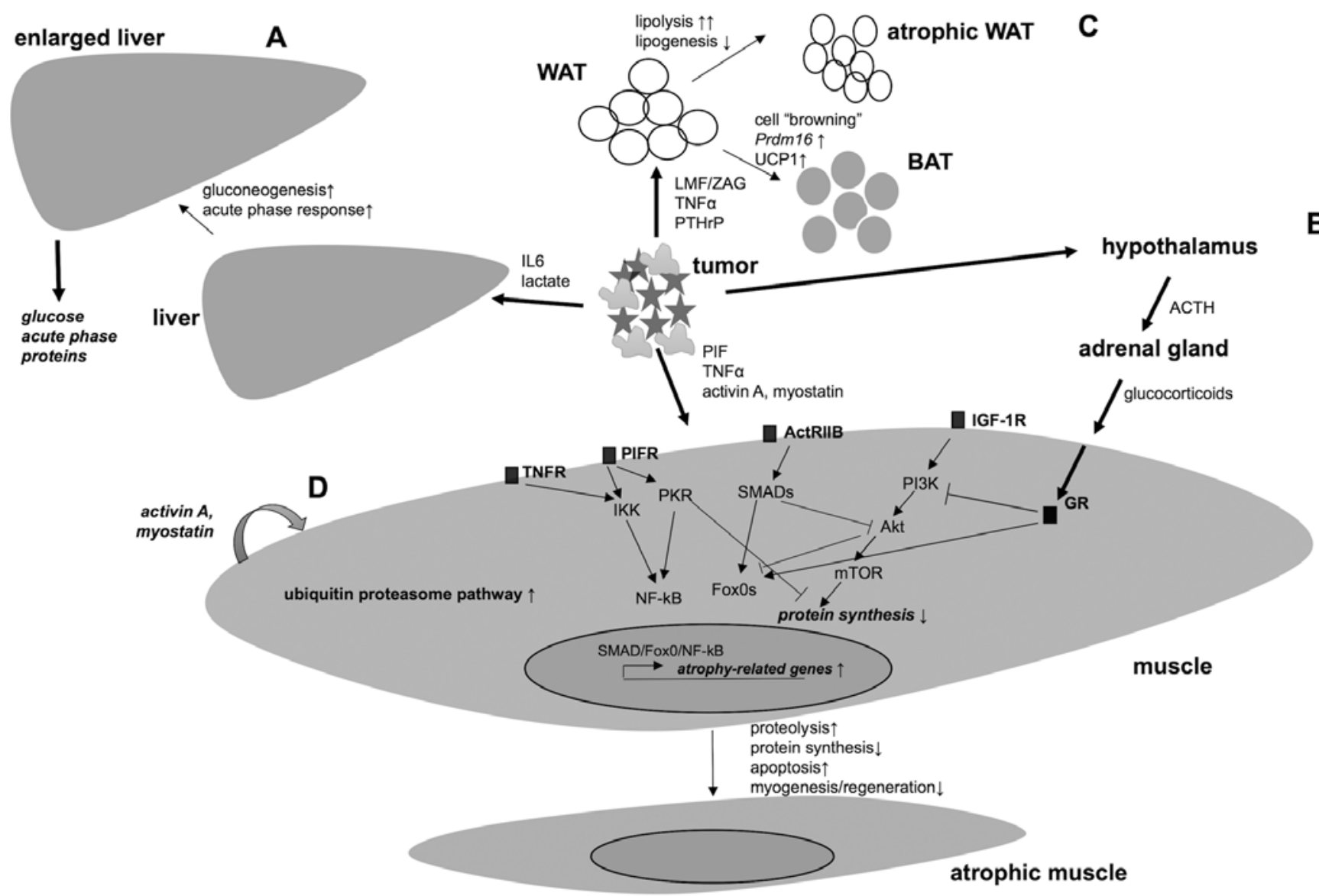

Figure 1. Cellular and molecular mechanisms of cancer cachexia, a muscle-centric view. Cachexia is the direct result of tumor growth and/or secretory activity, but may be the secondary effect of tumor control on bystander and/or target tissues. (A) Tumor-derived IL6 is a major elicitor of acute phase response APR, in addition lactate overproduced in intra-tumor aerobic glycolysis fuels hepatic gluconeogenesis leading to glucose overproduction. Liver in some cancer enlarges due to metabolic recycling activity - mainly Cori Cycle - and synthesis of acute-phase protein, in addition a chronic APR can exacerbate muscle wasting by increasing the demands for certain amino acids to support increased hepatic export protein synthesis. Finally, impaired liver metabolism contributes to high REE. (B) The tumor stimulates the HPA axis via inflammatory cytokines and noniceptive stimuli (not shown) leading to increased secretion of glucocorticoids by adrenal gland. (C) The tumor produces factors that reshape the metabolism of the adipose tissue towards catabolism and tissue shrinkage mainly via increased lipolysis. In addition, through PTHrP secretion, the tumor induces in WAT the expression of the Prdm16 master gene leading to cell 'browning'. Tumor-induced BAT dissipates energy via UCP1 contributing to high REE. (D) The tumor and bystander tissues produce a host of cachectic factors that reshape the metabolism of the muscle tissue towards catabolism and tissue shrinkage via increased proteolysis, decreased protein synthesis, increased apoptosis and decreased myogenesis/tissue regeneration. Besides IGF-1, the additional anabolic stimuli for muscle tissue are testosterone and physical exercise (not shown), all increasing the PI3K/Akt/mTOR axis and hence protein synthesis. Conversely the principal physiological catabolic control on muscle is produced by the TGF $\beta$-like myokines signaling via ActRIIB. During pathologic wasting the muscle catabolism is further driven by TNF and PIF receptors signaling activating NF- $\mathrm{kB}$ and PKR. Conversely ActRIIB signaling is independent from NF- $\mathrm{kB}$ activation. Finally, the wasting process may be driven by STAT3 activation whose principal elicitor probably is IL6 (not shown). Atrophy-related genes, termed atrogenes, constitute a common set of genes whose expression is coordinately induced or suppressed in muscle during the wasting process. $\rightarrow$ leading to activation, $-\mid-$ leading to inhibition, see text for further details.

humans. Moreover, a further increase in this factor has been associated with disease progression. Conversely, less is known presently about myostatin levels and activity in neoplastic diseases. Importantly, these factors may be secreted by the cancer cell or by its microenvironment, but tumors can also induce over-expression of myokines of the TGF $\beta$ family in the skeletal muscle, creating an autocrine loop (Fig. 1). At present these relevant points remain ill-defined and deserve further investigation (2,18 and references therein).

Myostatin and the other TGF $\beta$-like myokines bind to and activate a shared receptor-signaling apparatus, the activin receptor IIB ActRIIB. ActRIIB engagement gives rise to phosphorylation, assembly and nuclear translocation of transcription factors of the SMAD family, inducing an atrophy program. Moreover, signaling by TGF $\beta$-like myokines is tightly regulated by post-traslational and extra-cellular mechanisms. Myostatin circulates in the blood as a latent form which is activated via proteolisis, in addition, specific inhibitors such as follistatin can prevent binding of myostatin to ActRIIB. The existence of multiple control points emphasizes the importance of a tight regulation of TGF $\beta$-like myokines, in order to avoid muscle wasting and impaired muscle function $(2,18,20)$.

A comprehensive and in depth review of the mechanisms of cancer cachexia is beyond the scope of the present study, that focuses mostly on the atrophy of muscle and adipose tissues and on the high REE, this last issue being deeply interwoven with the altered metabolism in cancer. 
Atrophy of muscle tissue. Cancer patients with cachexia experience, nearly without exception, a progressive wearing down of skeletal muscle, which has suggested a muscle-centric view of this wasting syndrome (Fig. 1). Indeed, low muscle mass in advanced cancer is an independent predictor of poor prognosis and mortality (21). Skeletal muscle can undergo extensive remodeling that can cause some extent of physiologic and transient myofibrillar loss; indeed, normal homeostasis is a dynamic state resulting from a complex interplay of balancing forces (22).

The principal pathways regulating muscle tissue homeostasis are the insulin-like growth factor-1 IGF-1 receptor signaling pathway, that fosters hypertrophy via activation of the PI3K/AKT/mTOR axis and ActRIIB, that induces atrophy mostly via SMADs. During the wasting processes in cancer and other pathologic states, the balance may shift dramatically towards atrophy, due to diminished activity of hypertrophic pathways and/or increased ActRIIB activity.Pro-inflammatory cytokine receptor signaling can also contribute to atrophy by activating the IKK/NF- $\mathrm{kB}$ and/or JAK/STAT3 pathways (see below), as well as PIF-receptor signaling, that leads to atrophy via the IKK/NF- $\mathrm{KB}$ axis (Fig. 1).

Importantly, both decreased protein synthesis and increased proteolysis account for the net loss of myofibrillar proteins in the muscle cell. Three main proteolytic pathways, the autophagy/lysosome, $\mathrm{Ca}^{++}$-dependent calpains and the ubiquitin/proteasome-dependent proteolysis UPP, are responsible for protein catabolism (1) but in skeletal muscle, UPP is considered the most important in a range of catabolic conditions including severe sepsis and trauma, denervation atrophy, as well as cancer cachexia. Proteins intended for degradation by the proteasome are tagged by a covalently linked polyubiquitin chain in a multistep process involving the coordinated action of three enzymes: E1 (ubiquitin-activating enzyme), E2 (ubiquitinconjugating enzyme) and E3 (ubiquitin ligase). Indeed there are $>30$ different E2 and over $500 \mathrm{E} 3$ enzymes, which work in concert to confer exquisite substrate specificity to the UPP (23). Catalysis by specific E3 ligases is the rate-limiting step of the ubiquitination process, affecting the subsequent proteasomedependent degradation. Importantly, two E3 ligases, muscle RING finger-containing protein 1 (MuRF1) and muscle atrophy F-box protein (MAFBX, also known as Atrogin-1) are among the key factors induced via the atrophy pathways in order to cause structural muscle protein breakdown (1-3). For this reason, the over-expression of MuRF1 and/or MAFBX is widely used as signature of the wasting process.

A decreased rate of protein synthesis can be achieved by downregulation of the IGF-1/Akt /mTOR axis. Akt suppresses protein breakdown while promoting muscle growth by prompting the activity of the anabolic kinase mTOR and by inhibiting FoxO transcription factors, whereas, activation of ActRIIB causes Akt inhibition and FoxO activation. Finally, a depression of protein synthesis under catabolic stimuli in muscle may be the result of mechanisms acting at post-trascriptional level. In this context, a prominent molecular switch is increased in phosphorylation and hence inactivation of eukaryotic initiation factor $2 \alpha$ EIF- $2 \alpha$, a molecule required to begin translation (24). Both TNF $\alpha$ and PIF inhibit protein synthesis in muscle cells mostly through the phosphorylation of EIF2 $\alpha$ (see below) (1).
In summary, the overall outcome of atrophy pathways is the marked increase of nuclear accumulation of FoxOs and/or NF- $\kappa B$, leading, together with an increase in nuclear SMADs, to a rise of transcription of atrophy-related genes (atrogenes) (25) (Fig. 1), but also to a general suppression of myogenic programs, an aspect that will not be discussed.

Atrophy of adipose tissue. Cachexia is also characterized by loss of body fat, which seems to arise from an increase in lipolysis, rather than a decrease in lipogenesis (1), even if this view may be an oversimplification (3).

Loss of adipose tissue in cancer cachexia derives from massive hydrolysis of triglycerids and this increased lipid turnover results in increased plasma concentration of both glycerol and free fatty acids. As there is scarce evidence of cell death or necrosis, notion prevails that loss of fat mass is due to atrophy, rather than a reduction in adipocyte cell number. Finally, during cancer cachexia, white adipose tissue (WAT) undergoes a 'browning' process, in which it converts into cells resembling brown adipose tissue (BAT), also called beige cells (26). Further details on this important issue will be discussed below.

High resting energy expenditure. Approximately 50\% of cancer patients are hypermetabolic (REE $>110 \%$ of predicted) (27). REE is elevated in patients with both lung and pancreatic cancer, while there is no increase in REE in patients with gastric and colorectal cancer (1).

The pathways leading to hypermetabolism in cancer cachexia are varied and complex, and have been related to i) the peculiar cancer metabolism which is fueled by futile substrate cycles that occur when two metabolic pathways run simultaneously in opposite directions resulting in energy dissipation in the form of heat (28), ii) systemic inflammation and finally, iii) the presence of an elevated adrenergic drive (29).

It is well established that the main energy source in cancer cells is glucose, which is converted into lactate rather than $\mathrm{CO}_{2}$ (30), moreover some cancer cells display addiction to glutamine (31).

Cancer cells rewire cellular metabolism, partly because in the tumor microenvironment the oxygen may be insufficient to fuel the Krebs cycle, but mostly because aerobic glycolysis produces the anabolic drive that satisfy the high demands of fast growing cancer cells (30).

Accordingly, the lactate produced by cancer cells is transported to the liver and converted back to glucose and recycled back to the tumor. This is the Cori cycle, the representative futile cycle, whose overall effect is energy dissipation in the form of heat; indeed, the Cori cycle may account for a loss of energy in cancer patients of $300 \mathrm{kcal} /$ day $(1,32)$. Additional substrates, originated by lipolysis in adipose tissue or by protein catabolism in muscle tissue, also contribute to increased liver gluconeogenesis in cancer patients, but their contribution to increased REE is regarded as less important.

Increased expression of mitochondrial uncoupling proteins-1, -2, -3 (UCPs) is considered as a further common factor inducing hypermetabolism in cancer cachexia. The UCPs are a family of mitochondrial membrane proteins that mediate proton leakage and decrease coupling of respiration to ADP phosphorylation, resulting in the generation of heat 
instead of ATP (33). Therefore UCPs have been postulated to be major contributors in the control of energy metabolism. Of note, changes in expression of UCPs may be induced by tumor products or by cytokines that are produced during tumor progression. In support of this view, intravenous administration of LMF/ZAG to mice increases both UCP mRNA and protein levels in liver and skeletal muscle (34).

\section{A lesson from the field of infectious disease research, the utility of a measurement of tumor burden in the study of cachexia}

Actually a direct correlation between cachexia and tumor burden is the predicted outcome in cancer. The shrinkage of the tumor burden produced by surgery and/or anti-neoplastic therapies often leads to improvement in the cachectic state. Nonetheless, even within the same tumor type and burden, one individual may become cachectic whereas another will not. Such inter-individual variation may originate from the host genotype, as well as from other physiologic factors, such as diet, overall endocrine state or other less defined aspects of individual lifestyle. In addition and prominently, pharmacologic therapy may produce inter-individual variation in susceptibility to cancer cachexia.

In this frame in humans the genetic variation in the gene coding the MAP3K14 (NF- $\kappa \mathrm{B}$ inducing kinase NIK) is associated with different mortality in septic shock (35) and recent findings suggest a similar pattern in cancer cachexia. In other words, cachexia-prone genotypes may occur in natural populations, along with other genotypes that are more refractory to cachexia (36).

For example, in pancreatic cancer, $85 \%$ of patients become cachectic, but $15 \%$ do not. This is due to variation in tumor phenotype, but probably also to variation in host genotype (1).

Single-nucleotide polymorphisms in the IL-1, IL-6 and IL-10 genes that are linked to increased production rates of these cytokines have been associated in humans with the prevalence of cachexia in gastric or pancreatic cancer patients (2).

In conclusion, the progression of cachexia in cancer patients is not a totally tumor-dependent feature, but it is at least partially determined by the host response to tumor growth and spreading.

In recent times, a new concept has emerged in the field of infectious disease research that could be usefully applied to cachexia research $(37,38)$. Hosts that are good at reducing parasite burdens are not necessarily the healthiest. Stated in other words, hosts can sometimes be quite healthy despite high parasite burdens or conversely die with parasite loads which others survive. According to ecological immunology, the resistance to infection is defined as the potential of the host in restricting the pathogen load and this host feature is measured as the inverse of infection intensity (number of parasites/host or/unit host tissue). On the contrary, the ability of the host to endure the presence of the noxious agent, limiting the damage of a given parasite burden, is termed tolerance and it is operationally defined as the slope of a regression of host fitness or health state against infection intensity. The steeper the slope, the lower the tolerance Fig. 2A. Stated in other words, toler- ance is the rate of change in fitness or health state as parasite load increases.

The study of tolerance to parasites has a long tradition in plant science. For example, it has been established that plants with different genotypes may decrease their seed production at different rates in response to increasing pathogen load. In more recent times, these concepts have also been applied to the field of animal infectious diseases. As one of many possible examples, mice deficient in the anti-inflammatory cytokine IL-10 exhibit much higher mortality rates than wild-type mice in the presence of similar loads of Plasmodium chabaudi (39). Thus, in this case there was no difference in resistance, but IL-10 knockouts nonetheless suffered more from the infection.

Nowadays, the overall picture suggests that genes involved in tissue repair and scavenging of damaging molecules produced during infection, as well as factors regulating immune responses, are prime candidates for playing a pivotal role in host tolerance to infectious diseases.

Importantly, Medzhitov et al (40) claim that the concept of tolerance to infections is applicable to any disease associated with tissue damage, stress, malfunction or loss of homeostasis. Along these lines, the aim of the present article is to investigate the cancer disease in an ecological perspective, where the host response to the tumor growth and metastatic spreading is the wasting process known as cachexia.

We highlight the relevance of introducing the plot analysis of informative correlates of disease progression vs. the tumor burden, in the present study defined as the aggregate of the primary tumor mass and metastasis masses, for a better assessment of cachexia. In the legend of Fig. 2A and B two proposed informative correlates of disease progression - health state $H S$ and cactectic index $C C$ - are described in depth.

Introducing measurement of tumor burden may allow i) to assess inter-individual variation in cachexia, ii) to evaluate whether drug treatments reducing the overall tumor burden act independently on the host producing an amelioration of cachexia. In this case, improvement of cachectic state is not a mere side effect of decreased tumor burden and finally iii) to define which, among the host defense mechanisms and therapeutic effects, are involved in restriction of tumor burden and which limit damage to the host.

In conclusion, the liaison between tumor burden and cachexia is fairly complex and an investigation of this relationship is the aim of the present study.

\section{Experimental oncology, rodent models}

Several studies aiming at preventing or slowing down the development of cancer-induced cachexia have been published using murine models. To this end, cancer was induced and the development of cachexia was monitored comparing genetically engineered mice with their wild-type counterparts or mice with different endocrine backgrounds or mice receiving different drug treatments. In most cases, the experimental condition under investigation did not affect food intake ruling out the possible interference of anorexia in the wasting process Table I and II.

Actually the resulting changes in the wasting process define two possible scenarios: i) improvement in the general health state parallels the decrease in overall tumor burden or 


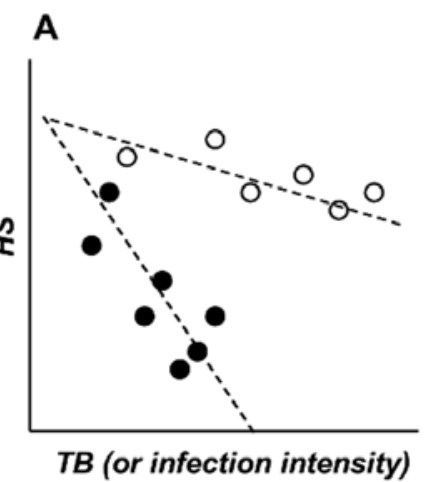

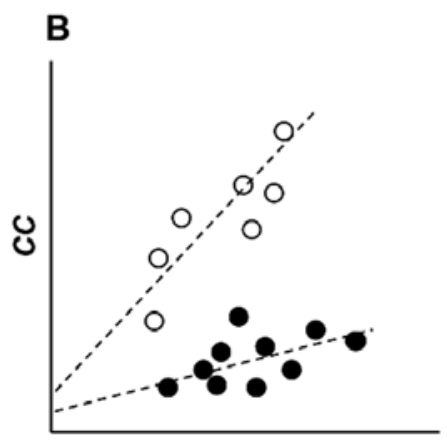

$T B$

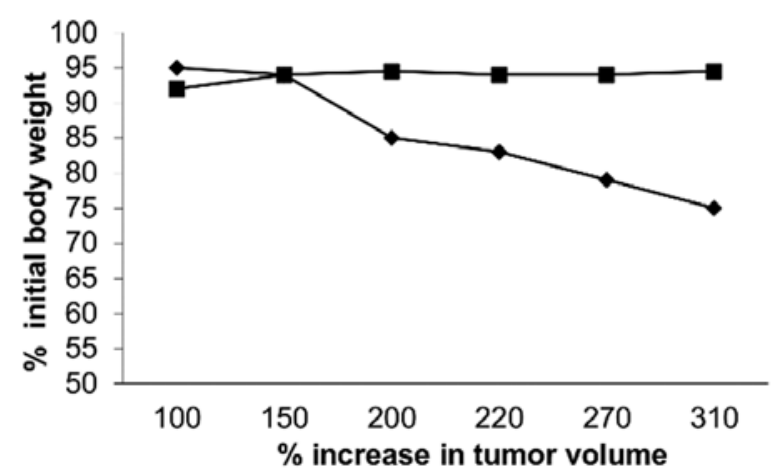

Figure 2. Plot analysis of informative correlates of disease progression vs. the tumor burden. Group A (open circles) and group B (filled circles) refer to individuals that differ for some biological feature genotype, therapy, gender, impacting the course of the cancer-induced wasting process: (A) the physiological parameters of cancer progression that tend to decrease with worsening of prognosis pertain to health state HS; examples are: percentage loss of initial body weight or muscle mass, albuminemia. HS is plotted vs. tumor burden TB. Of note, there is no a priori reason to assume that the relationship between the host HS and the TB should be linear (redrawn with modifications after Raberg et al (38) Fig. 1 portraying two host genotypes that perform differently in a fitness $v s$. infection intensity plot). (B) The physiological parameters of cancer progression that tend to increase with worsening of prognosis may be regarded as correlates of cachexia $C C$. Informative $C C$ include blood markers produced by host tissues rather than by the tumor, examples are: serum concentration of $\mathrm{CRP}$ or serum concentration of metabolites such as fatty acids. Conversely the blood analytes produced mostly by the tumor mass are not bona fide $C C$ since they rest on tumor burden. CC is plotted vs. tumor burden TB. (C) To increase the statistical significance of the cachexia evaluation, the ratio between HS and TB should be analyzed during the temporal progression of the neoplastic disease in an $H S / T B$ vs. time plot (or in a $C C / T B$ vs. time plot, not shown). That is, the plot analysis shown in Fig. 2A is reiterated and each point in Fig. 2C represents the average value of HS/TB ratios in group A or group B \pm error bars for each chosen time point. (D) Analysis in an $H S$ vs. TB plot of data from Beck et al (51). Mice bearing MAC16 adenocarcinoma treated with EPA (filled squares) or mock-treated (filled diamonds). In the original report the percentage loss of initial body weight and the percentage increase in tumor volume were both plotted separately against the days of dosing.

ii) improvement in the general health state is basically independent of the overall tumor burden.

i) Inhibition of cachexia associated with a decrease in tumor burden. It is well established that neoplastic progression and inflammation are deeply interwoven.

As a matter of fact, the current literature highlights a role for chronic inflammation in virtually all the steps of tumorigenesis, including tumor initiation, promotion and progression (41). In addition the cancer-related wasting process is driven by inflammation as well. Significantly, nearly all the experimental systems, where an alleviation of cachexia with parallel decrease of the tumor burden was observed, have been produced by prompting in the host a reduction of inflammation (Table I).

According to the literature the most rewarding pharmacological targets for reduction of cachexia are $\mathrm{NF}-\kappa \mathrm{B}$, the ubiquitin-proteasome pathway UPP and the protein kinase dsRNA-dependent PKR.

Remarkably, NF- $\kappa \mathrm{B}$ and the UPP are deeply interconnected through positive-feedback circuits, indeed the proteasome function is required for the activation and nucleus migration of $\mathrm{NF}-\kappa \mathrm{B}$, conversely in the nucleus $\mathrm{NF}-\kappa \mathrm{B}$ binds and transactivates the promoters of proteasome subunit coding genes (42). Moreover, a positive feedback loop exist also between $\mathrm{NF}-\kappa \mathrm{B}$ and inflammatory cytokines, such as $\mathrm{TNF} \alpha$, since signaling downstream of these cytokines induces $\mathrm{NF}-\kappa \mathrm{B}$ activation, while NF- $\kappa \mathrm{B}$ modulates the expression cytokine-encoding genes (43).

Targeting $N F-\kappa B$. NF- $\kappa \mathrm{B}$ is known to be constitutively activated in certain cancer cell lines, resulting in enhanced cell survival and proliferation, as well as in in vivo tumors, where it also supports angiogenesis and invasion (43). At the host level, activation of $\mathrm{NF}-\kappa \mathrm{B}$ has been shown to cause muscle atrophy via accelerated protein breakdown, by increasing the expression of key components of the UPP (such as proteasome subunits and the MuRF1E3 ligase) (44). Noticeably NF- $\mathrm{B}$ activation in muscle results mainly involved in the 'pathological' pathways of degradation or remodeling of myofibrillar proteins as induced by $\mathrm{TNF} \alpha$ or tumor-derived PIF. In fact $N F-\kappa B$ in muscle is activated by disuse (45) or sepsis (46). Contrariwise, 


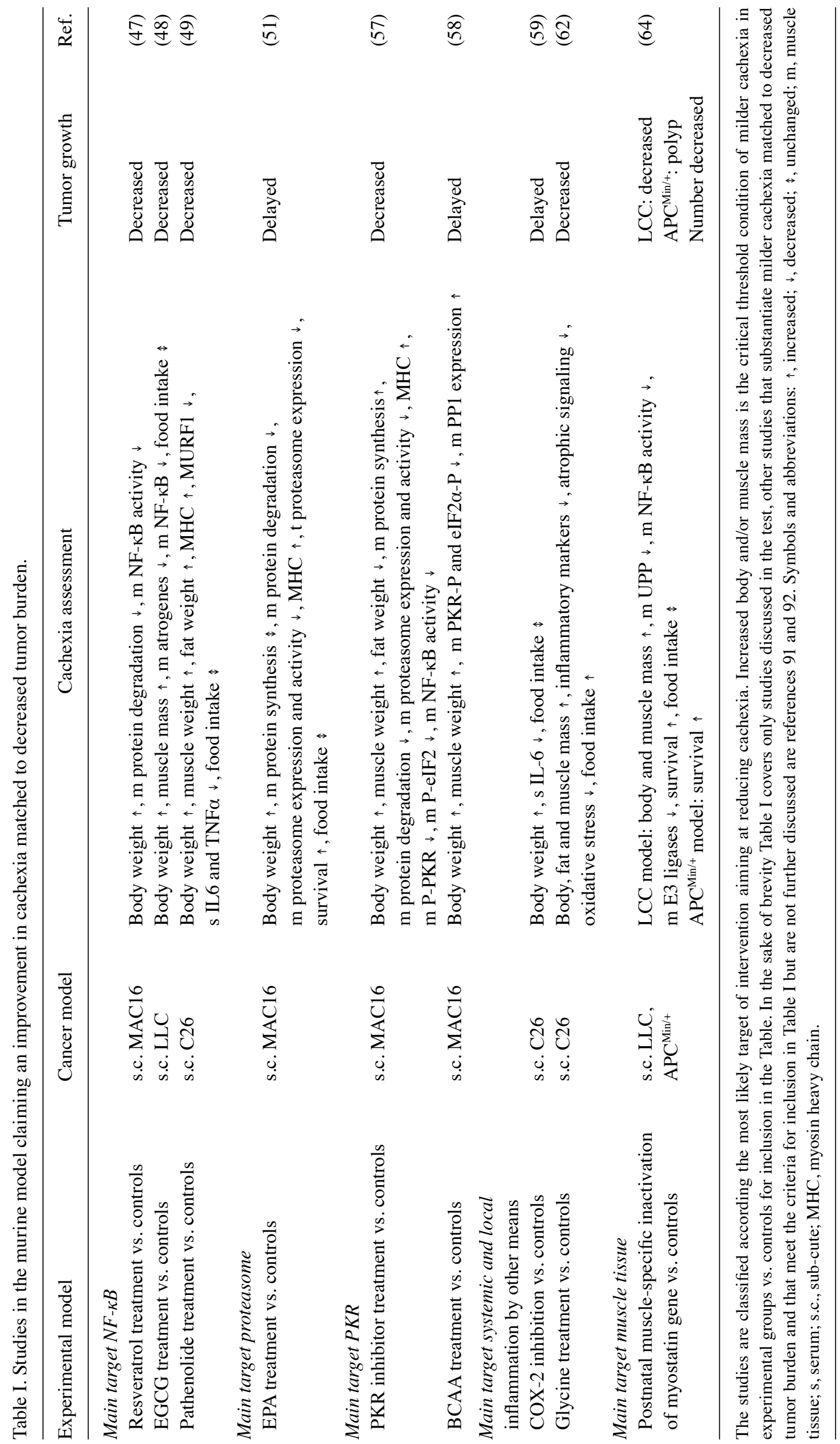




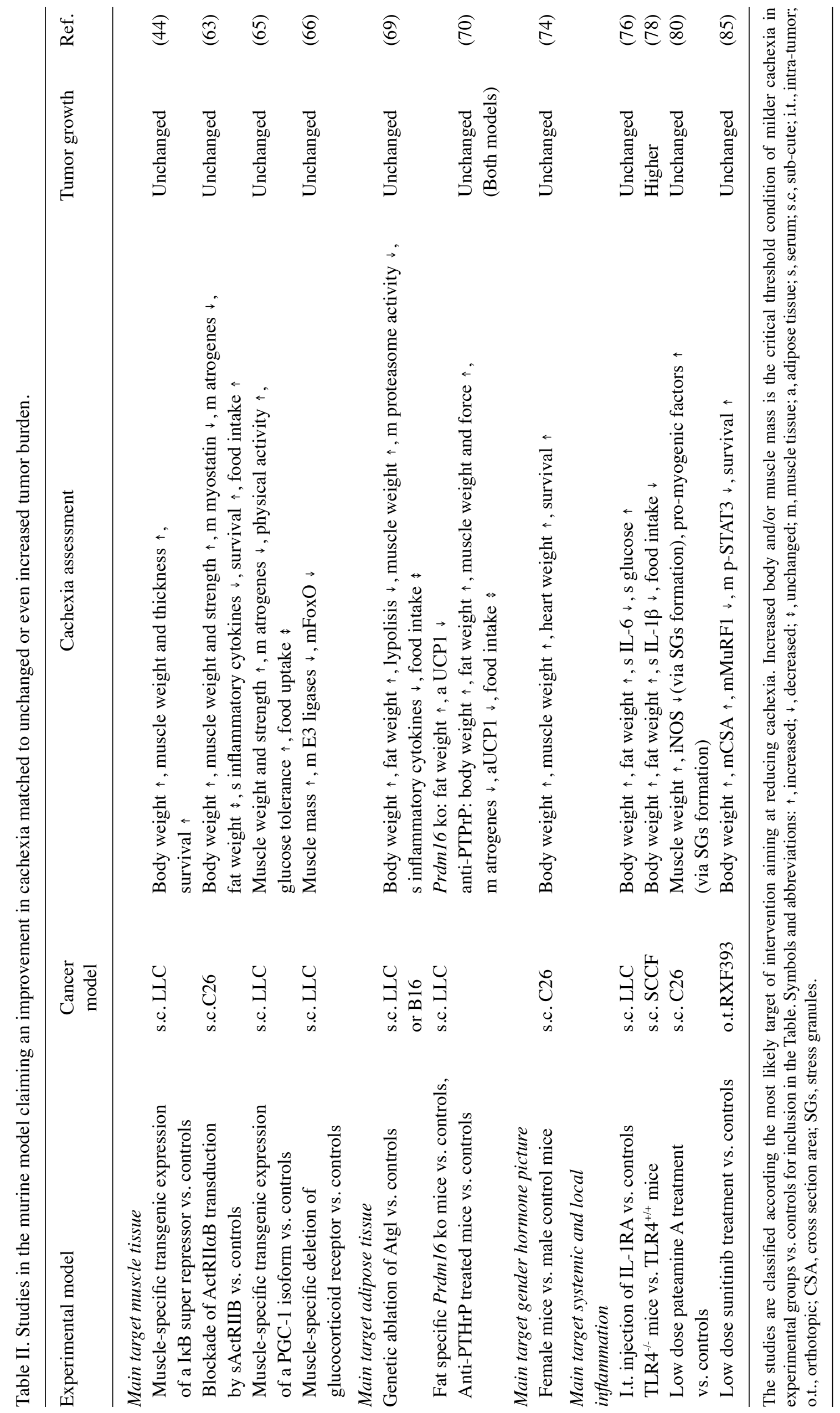


the 'physiologic' pathway of muscle atrophy under signaling by TGF $\beta$-like myokines is instead NF- $\mathrm{BB}$-independent $(2,20)$.

Resveratrol, parthenolide and epigallocatechin-3-gallate are well known nutraceutical compounds that alleviate inflammation through inhibition of IKK, the upstream kinase that target $\mathrm{I} \kappa \mathrm{B}$ for destruction in the UPP, releasing active $\mathrm{NF}-\kappa \mathrm{B}$. These three drugs have been investigated in an attempt to alleviate the cancer-induced wasting syndrome. In fact the drug treatment attenuated weight loss and produced a significant improvement in skeletal muscle by dampening $\mathrm{NF}-\kappa \mathrm{B}$ activity in this tissue while decreasing tumor growth. Moreover, the effect on tumor burden was anticipated since these phyto-pharmaceuticals target both cancer cell growth and tumor microenvironment through inhibition of $\mathrm{NF}-\kappa \mathrm{B}$ activity (47-49).

Targeting UPP. The proteasome pathway nowadays is investigated as a promising molecular target for cancer therapy due to its pivotal role in $\mathrm{NF}-\kappa \mathrm{B}$ activation. Moreover, most cancer cells are highly proliferative and have an increased requirement for protein synthesis, which makes them highly exposed to UPP-driven quality control for protein synthesis and misfolding (50).

Eicosapentaenoic Acid EPA directly affects the expression of proteasome subunits, rather than acting as a direct inhibitor of proteasome activity. This substance produces a significant delay in tumor growth while attenuating weight loss in mice bearing the cachexia-inducing MAC16 adenocarcinoma $(51,52)$. The inhibition of tumor growth was expected since PUFAs of the $\omega-3$ series have been shown to decrease growth in murine cancers (51).

A major concern in the present study of cachexia is to rule out that the improvement of health state is the mere consequence of tumor debulking. With regard to this point, the introduction of tumor burden assessment may be highly informative. This is the case of EPA therapy since mice bearing a large tumor burden are still alive, while control mice succumb to a much lower tumor burden. This fact is much clearer by plotting body weight against tumor burden Fig. 2D, as also applied previously (53).

The above plot analysis highlights that EPA treated mice are subjected to a lower decay rate of health state in comparison to control mice. This represents the more convincing evidence that the treatment elicits a response on the host distinct from the direct antitumor effect.

Targeting PKR. A further much investigated target for attenuating cancer cachexia is PKR (54). This inducible sentinel kinase and stress sensor blocks global protein synthesis through eIF $2 \alpha$ phosphorylation, leading to cell death in response to a variety of cellular stresses. However, PKR also activates $N F-\kappa B$, promoting cell proliferation. Due to this dual function, the role of PKR activation in cancer remains controversial. Indeed, since clinical data show a correlation between suppressed or inactivated PKR and a poor prognosis for several cancers, PKR is considered to have a tumor suppressor function. Conversely, other evidence supports the view of PKR activity inhibition as a likely objective for cancer therapy. This latter point holds true for the wasting response in host tissues to the cancer aggression. Two pro-cachectic factors, PIF and angiotensin II, act inducing autophosphorylation and activation of PKR in muscle cells (55).

Furthermore, activation of PKR also induces protein degradation in muscle through the induction of the expression and activity of UPP in an NF- $\mathrm{BB}$-mediated process (55). Finally, growth factors such as IGF-1, which attenuate protein degradation in muscle, also counteract the activation of PKR (56).

In tumor-bearing mice a specific inhibitor of PKR effectively attenuates the depression of body weight, increases muscle mass and inhibits tumor growth (57). In addition, the muscle is a likely direct target of the treatment since in this tissue the rescue of protein synthesis and normalization of the biochemical markers related to activity of PKR is observed. Interestingly, the improvement in health state is not the mere consequence of shrinkage of tumor mass since the former precedes the latter by several days, a further example of the importance of a measurement of tumor burden in the study of cachexia. As underlined above, the decreased tumor growth by PKR inhibition is somewhat unexpected since PKR has been suggested to act as a tumor suppressor gene. An explanation may be that the PKR inhibitor impairs NF- $\kappa \mathrm{B}$ activity in the tumor microenvironment as it does in skeletal muscle. Another possible explanation is that the tumor requires amino acids released from skeletal muscle during proteolysis for growth (57). This last scenario is an interesting inverse perspective since it is usually acknowledged that tumor debulking drives an improvement in health state not the reverse.

Another approach under investigation for inhibiting PKR in muscle tissues is the therapy with branched-chain amino acids BCAA, since these amino acids function not only as building blocks, but also as modulators of protein synthesis. In particular, in vivo leucine significantly attenuates in muscle the increased phosphorylation of PKR and eIF $2 \alpha$ induced by tumor growth and this effect is associated to the increased expression of protein phoasphatase-1 PP1. Even in this case the improvement of cachexia is accompanied to a lower tumor burden (58).

Targeting systemic and local inflammation by other means. Celecoxib is a specific inhibitor of COX-2. This drug was clearly able to delay tumor growth in mice engrafted with C26, but was also very effective in controlling tumor-induced wasting even in animals carrying high tumor burden that proved lethal in control mice (59). The inhibitory effect of celecoxib on tumor growth is expected since COX-2 is known to be over-expressed in tumors leading to aberrant angiogenesis and cancer cell proliferation (60). On the other hand, the impact of celecoxib in reducing the wasting syndrome may be related to a decreased production by the tumor of cachectic factors, mainly pro-inflammatory cytokines.

Above the modulation of protein synthesis amino acids may tune other physiological processes and this is the case of glycine that acts on inflammation producing inhibitory effects on several white blood cells. Indeed, dietary glycine is protective in rat models of endotoxemia, liver ischemia reperfusion and liver transplantation through reduction of serum TNF $\alpha$. The mechanism is the activation in inflammatory cells of glycine-gated chloride channels, thereby effectively reducing calcium influx in these cells (61). In C26 engrafted mice, glycine administration protects skeletal muscle from 
cancer-induced wasting and loss of function, reduces the oxidative and inflammatory burden and reduces the expression of genes associated with muscle protein breakdown in cancer cachexia. Even in this case these effects are accompanied by an inhibition of tumor growth (62).

ii) Inhibition of cachexia disentangled from the tumor burden. In this section, we seek to discuss the studies in which the inhibition of cachexia is not accompanied to a decrease of tumor burden. In this circumstance the tumor burden remains fairly unchanged, actually in one case amazingly increases in spite of the amelioration in the wasting disease Table II.

In the present study, the interference with cancer-induced wasting syndrome more frequently is the result of a local, rather than systemic change in physiology or the outcome of a pharmacological therapy targeting the tissues directly involved in cachexia. The main focal point is at variance with the muscle or the adipose tissue and it is interesting to note how the local improvement in physiology often produces a positive effect on the whole organism irrespectively of the tumor burden that remains unaffected.

Recent evidence suggest that the host genotype may affect the course of cachectic wasting, indeed this outcome may be experimentally produced by tissue-specific targeted transgenesis. Actually, this reductionist approach constitutes an informative case study for future investigations on the genetic variation affecting the vulnerability to cancer cachexia due to allelic polymorphisms present in natural population in both mice and humans.

Targeting muscle tissue. As a matter of common knowledge, the muscle is regarded as the principal target tissue of cachexia. In an attempt of changing the local response to wasting stimuli coming from the tumor growth, the $\mathrm{NF}-\kappa \mathrm{B}$ function was disrupted by introducing the expression of a dominant inhibitory I $\mathrm{KB}$ in skeletal muscle but not in the rest of the organism. By this means a clear improvement in the health state in tumor bearing transgenic mice was observed vs. controls in spite of the similar tumor burden (44).

A second approach focused on muscle was to counteract the catabolic effect of myostatin and other myokines of the TGF $\beta$ family by administration of an ActRIIB soluble decoy receptor, since ActRIIB functions as the common receptor for both myostatin and activins. Remarkably, the treatment with the ActRIIB trap blocked cachexia in the C26 model of cancer cachexia without effecting tumor growth. In addition, the present study clearly shows that preserving muscle mass is of major importance in determining organism's survival $(53,63)$. In truth, in relation to this latter point, to date the overall context is rather contentious. Vulnerability to cachexia ensuing from tumor engraftment in mice devoid of myostatin from the earliest stages of development increases as reported by Klimek et al (53), but instead decreased in the study by Gallot et al (64). Furthermore, in this latter study the improvement in cachexia due to lack in myostatin signaling correlates with a decrease in tumor burden (Table I), whereas others $(53,63)$ reported that the amelioration of cancer cachexia obtained through the acute inhibition of ActRIIB signaling is not accompanied to a decrease of tumor burden (see below for further discussion).
A third approach focused on muscle is based on PGC-1 $\alpha$, a transcriptional coactivator induced by exercise that gives muscle adaptation to endurance-type exercise. Mice with skeletal muscle-specific transgenic expression of PGC-1 $\alpha 4$, an alternative splice form highly expressed in exercised muscle, show increased muscle mass and strength in control mice and dramatic reduction of vulnerability to muscle atrophy in tumor-bearing mice. Even in this case, the tumors in both transgenic and control mice grow at the same rate (65).

Mice with targeted ablation of the glucocorticoid receptor in muscle mGRKO were proven less vulnerable to muscle atrophy in response to tumor growth. Even in this case, tumors grew to equivalent sizes in mGRKO and control mice (66). The classical effects of glucocorticoid signaling depend on GR-mediated transcription and hence de novo protein synthesis but emerging evidence suggests that glucocorticoids can also exert their actions through more rapid signaling mechanisms that do not require nuclear GR-mediated transcription. Therefore, the GR engaged with its ligands may affect the signaling state of key cytosolic transducers (67). In the muscle environment, glucocorticoids appear to directly downregulate the PI3K/Akt pathway by several post-transcriptional mechanisms. In particular, increased expression of FoxOs by glucocorticoids activates the gene transcription program leading to muscle atrophy Fig. 1 (68).

Targeting adipose tissue. A further promising target of intervention in order to counteract cachexia is the fat tissue. Actually, the marked depletion of adipose mass in advanced cancer may arise from either a decrease in the synthesis of triglycerides or an increase of lipolysis even if the latter is widely regarded as the principal mechanism. Inhibition of lipolysis through genetic ablation of adipose triglyceride lipase Atgl, a gene predominantly expressed in adipose tissue, ameliorates certain features of cancer-associated cachexia. In fact tumor bearing Atgl deficient mice resist increased WAT lipolysis, but also myocyte apoptosis and proteasomal muscle degradation thereby maintaining normal adipose and muscle mass. Tumor weights tend to be lower in $\mathrm{Atgl}^{-/-}$mice than in control mice but these differences do not reach statistical significance (69). The present study suggests that pharmacological inhibition of metabolic lipases may help prevent cachexia.

One key characteristic of cancer-induced cachexia is the higher REE level than in healthy animals. Brown fat dissipates chemical energy in the form of heat and hence, may be involved in the negative energy balance in cancer. How tumors induce thermogenesis in brown fat cells and how this may relate to the wasting of fat and skeletal muscle has emerged in recent years. In normal physiology white fat depots contain nests of uncoupling protein 1 (UCP1)-expressing multilocular cells, called beige cells. These cells can be induced or further stimulated on exposure to cold or other stimuli via a process termed browning and the transcriptional coregulator PRDM16 is the master regulator in the browning process (70) (Fig. 1).

Fat-specific Prdm16-deficient mice, which have a dramatically reduced thermogenic potential and are resistant to induction of browning, are less vulnerable than control mice to adipose tissue loss if challenged with tumor growth. This better outcome is accompanied to a reduced loss of muscle mass and occurs without a change in tumor size in comparison to control 
mice (70). Further investigation in this experimental model has shown that tumor-derived parathyroid hormone-related protein (PTHrP) (Fig. 1) has an important role in wasting, through driving the expression of genes involved in the browning of the adipose tissues. Neutralization of PTHrP in tumor-bearing mice blocks adipose tissue browning and the loss of muscle mass and strength, even in this case without a reduction of tumor burden (70). Thus, neutralization of PTHrP may hold promise for ameliorating cancer cachexia and improving patient survival.

In conclusion, these studies highlight the fact that physiologically important crosstalk between adipose tissues and skeletal muscles does exist in the context of cancer cachexia. Therefore a therapeutic endeavor on skeletal muscle is expected to produce a better outcome on adipose tissues and also the reverse is supposed to hold true.

Targeting different endocrinology between experimental groups. A significant gender dimorphism has been observed in muscle mass loss and survival among cancer patients. In fact, multiple studies have found that male cancer patients lose more body weight and muscle mass than females and have a shorter overall survival rate $(71,72)$. It is likely that gender hormones are mediating these differences because postmenopausal females lose their survival advantage and estrogen therapy decreases colon cancer mortality (73).

In this frame, male tumor-bearing mice display a more severe phenotype than female, including greater body mass loss and mortality and a more robust pro-inflammatory response. The decreased vulnerability to cancer cachexia in females is due to estrogen signaling as assessed by in vivo treatment with a specific estrogen receptor antagonist. Moreover, this gender dimorphism cannot be attributed to differences in tumor growth (74).

Remarkably, a major contribution to differences in cachexia between genders is due to cardiac atrophy and impaired function. Cardiac atrophy is mainly due to the autophagy/lysosome pathway, a mechanism by which cells degrade large quantities of intracellular protein during periods of cellular stress. Of note the autophagy/lysosome pathway has been shown to play a greater role in the heart than in skeletal muscle $(74,75)$.

Targeting systemic or local inflammation. In the previous section several studies has been discussed where the restraint of systemic inflammation produces an improvement of cachexia while decreasing tumor burden via somewhat related mechanisms. In the present study, evidence are shown that dampening of inflammation in the whole animal or in the tumor microenvironment may even produce an improvement of cachexia not paralleled by lessening of tumor burden.

Indeed this is the case of intratumoral injection of IL-1 receptor antagonist IL1RA that reversed weight loss in C26-bearing mice without affecting the rate of tumor growth. Moreover, this treatment improved lean and fat tissues, as well as hypoglycemia and serum IL-6 level. These results suggest that, at least in the $\mathrm{C} 26$ model, tumor produced IL-1 acts on the local induction of cachectic factors rather than by implementing tumor growth (76).

A second intriguing report concerns TLR4. This the well-known pattern recognition receptor (PRR) binds to lipopolysaccharide LPS from gram-negative bacteria and to other pathogen associated molecular patterns (PAMP), in addition TLR4 signals the over-presence of numerous endogenous damage associated molecular patterns (DAMP) (77).

An inbred mouse strain homozygous for a non-functional TLR4 is protected from cancer-induced wasting in spite of an increased tumor growth in comparison to wild-type control mice $(78,79)$.

Therefore, in absence of TLR4-driven signaling the growth of cancer is subjected to lesser restrictions, but the host is less vulnerable to cachexia. This surprising and counterintuitive evidence awaits further investigation.

As a further attempt at counteracting cancer cachexia, low dose panteamine A prevented the tumor-induced loss of skeletal muscle in mice without affecting tumor growth (80). Panteamine $\mathrm{A}$ at relatively high concentration inhibits general translation, inhibits cancer cell proliferation and is toxic (81). Conversely at low doses this drug counteracts tumor-induced cachexia by blocking in the muscle the expression of the pro-cachetic factor iNOS, while rescuing the expression of pro-myogenic factors myoD and myogenin. In fact, pro-inflammatory cytokines over-produced in cancer stimulates muscle cells in an NF- $\mathrm{NB}$-dependent manner, and the expression of iNOS in turn promotes muscle wasting $(82,83)$. The differential effect on pro-cachectic and pro-myogenic factors is due to the fact that low-dose panteamine A, by selectively acting on iNOS mRNA, promotes its recruitment to stress granules SGs (84), where this transcript remains in a translationally repressed state. Conversely, in response to panteamine $\mathrm{A}$, the MyoD and Myogenin mRNAs are not recruited to SGs, but rather associate with heavy polysomes, which leads to the rescue of their expression.

Finally, sunitinib has been recently employed to prevent cachexia in nude mice bearing an orthotopic xenograft of human renal carcinoma (85). Sunitinib is a tyrosine kinase inhibitor mainly targeting VEGF signal transduction and hence tumor angiogenesis. This drug has shown therapeutic efficacy in renal cell carcinoma in humans, but in this case the increased overall survival rate is not fully explained by the antitumor activity of the drug. Indeed in humans sunitinib frequently induces disease stabilization rather than regression (85). In the murine model this kinase inhibitor dramatically decreases the host vulnerability to tumor growth and in addition low-dose sunitinib counteracts cachexia without exerting antitumor effects. As claimed (85), in tumor-bearing animals sunitinib acts on skeletal muscle as a consequence of the inhibition of STAT3 activation and of MuRF1 over-expression. JAK/STAT3 pathway likely plays a relevant role in muscle wasting even though in this case the mechanisms of atrophy induction are still ill-defined (86).

\section{Cancer in humans: biased, hypothesis driven and unbiased approaches to the study of cachexia}

Whereas in experimental oncology the analysis of cancer cachexia and its progression in relation to host genotype or pharmacological treatment has been coupled with the measurement of tumor burden, in clinical oncology a similar analytical approach has not been ventured yet.

Possibly this delay is due both to the difficulty of acquiring informative measurements for cachexia and tumor burden in humans and to the actual lack of interest in clinics. 
On the other hand, the experience in the research field of infectious diseases calls attention to the potential interest for investigations in basic oncology of plotting, even in humans, quantitative correlates of cachexia against the tumor burden.

An in depth discussion in clinical oncology of the pitfalls in the quantitative evaluation of cancer-induced wasting syndrome and of in vivo imaging for measurement of tumor burden is beyond the scope of this exploratory report. We shall single out here only that an overall continuous, numerical measurement of cachexia is likely misleading since this syndrome results from a complex array of symptoms. Moreover, cancer cachexia is a continuum with three stages of clinical relevance: pre-cachexia, cachexia and refractory cachexia (5). The aim of a consensus clinical definition of cachexia is approaching a classification procedure for this condition, akin in some respects to the TNM staging system used in clinical oncology. On the other hand, in order to solve this issue, the separate anatomical, functional or biomolecular correlates of the wasting syndrome may be plotted one by one vs. the tumor burden.

On the understanding that the main conclusion of plotting cachexia against tumor burden should be a better evaluation of the inter-individual variability in the vulnerability to cachexia, our interest is to summarize briefly two potential procedures to this analysis.

In the biased, hypothesis driven approach, patients homogeneous for cancer diagnosis and therapy protocol are recruited on the basis of a previously established rational and placed in two groups according to distinct biological or clinical features. The hypothesis of the study is that the two groups perform differently in the cachexia vs. tumor burden plot. That is, the patients enrolled in the study may be grouped on the basis of a known genotype, a different pharmacological treatment, diet, other habits or physiological features. Examples of use of cachexia/tumor burden plot are the comparison of male vs. female patients or lean vs. overweight patients. In relation to the latter example, it is interesting to remark that overweight/obesity is a risk factor in developing cancer disease but constitutes a better prognosis factor in advanced disease. Indeed this is known as the obesity paradox (87).

In the unbiased approach the patients recruited in the study are analyzed in the cachexia/tumor burden plot on the basis of a shared cancer diagnosis and therapy protocol, but irrespectively of any hypothesis on cachexia. This approach would be a discovery tool to identify a minor subset of patients differently placed in the cachexia/tumor burden plot. Moreover, in order to add significance to the present study, this evaluation should be carried out prospectively for the ensuing time-points (Fig. 2C). The final goal of this type of study is to unravel in functional and molecular terms, for example by means of genome-wide approaches, the key differences between the divergent groups of patients in the cachexia/tumor burden plot that may possibly arise from the unbiased approach.

\section{Discussion}

The present study investigates cancer cachexia mostly within experimental oncology on murine models. Conversely the significance and feasibility of introducing a measurement of tumor burden for a better assessment of cachexia in clinical oncology is just preliminarily outlined.
As shown in the murine model, a significant recovery from cancer cachexia due to variation in host biology may match with decreased, unchanged or even increased tumor burden (Table I and II). A major point of discussion is to unravel this heterogeneity. On this subject it is tempting to speculate that when the change in host physiology associated to milder cachexia is confined to a tissue, direct target of the wasting process, the tumor growth proceeds unrestricted. On the other hand, the result on tumor growth of a systemic change in host physiology leading to milder cachexia is likely more variable and hence controversial. Here usually the improvement in cachexia is accompanied to a decrease in tumor burden, but it is not always the case. Indeed inflammation sometimes may produce a dual effect on the cancer cell and its microenvironment, on one side, and on host physiology, on the other side. For example, inflammation could restrict tumor growth while promoting the wasting process in the host 'periphery'. This intriguing scenario may be suggested by the outcome of tumor challenge in mice devoid of TLR4 expression $(78,79)$.

Distinct subclones of the same tumorigenic cell lines may produce in vivo striking different outcome in host cachexia (19,88-90). For example, Pten $^{+/} \mathrm{E} \mu$-Myc lymphoma, in contrast to parental $\mathrm{E} \mu$-Myc lymphoma, produces the cardinal features of cachexia (89). Of note, even in this case, cachexia and tumor burden result disentangled since better outcome and survival is accompanied to increased growth of neoplasia (89). These interesting studies are not further discussed here since the subject of the present article is the variation in cachexia due to host biology rather than to tissue-intrinsic features of the cancer cell and/or of the tumor microenvironment.

Within the experimental study of cachexia in mice, until now the most popular approach has been a reductionistic model of malignancy based on the engraftment of highly cachectic cell lines such as LLC, C26, B16 and MAC16. In spite of the interesting published results, this field of investigation is in its infancy even because the more informative experimental models today available, transgenic mice strains developing tissue-specific spontaneous neoplasia or the orthotopic engraftment of tumorigenic cell lines, are been employed rarely in these studies. An exception is the well established $\mathrm{APC}^{\mathrm{Min} /+}$ mouse developing intestinal polyposis $(64,91,92)$. In most cases, the assessment of tumor burden has been performed only in the site of engraftment of the pro-cachectic cell line measuring by a caliper the dimensions of the tumor explant. Accordingly, there is plenty of room for improvement through employment of highly informative imaging techniques today available for the analysis of malignancy in mice (93).

Several issues broadly related to the relationship between cachexia and tumor burden remain incompletely understood needing further investigation.

i) when the drug treatment results in a tumor burden debulking matched with milder cachexia, the question is how to ascertain that the latter is not the mere aftermath of the former. As shown in the present study, in many cases a careful time-course measurement of tumor burden would contribute to the solution of this conundrum. A further procedure is inducing the wasting process in a way that mimics the cachectic diseased state in cancer in a tumor-free environment, for example by injection of pro-inflammatory cytokines in the bloodstream (80). 
ii) another relevant issue is a careful evaluation of the effect of drug treatment on the in vivo tumor vs. the in vitro cancer cells. Indeed a decreased tumor mass after treatment does not imply a direct cytotoxic or cytostatic effect on the cancer cells, contrariwise an in vitro cytotoxic effect on the cancer cells does not result, without exception, in in vivo decreased tumor mass. An example is the amino acid glycine that increases in vitro cancer cell proliferation (94) while in vivo impairs tumor growth through inhibition of angiogenesis $(62,95)$.

iii) several molecules produced within the tumor mass or by the cancer cell itself enforce cachectic wasting at variance by acting on skeletal muscle-myostatin, activins, PIF, or on adipose tissue, LMP/ZAG and PTHrP (Fig. 1). It is worth to note that, whereas the impact of these factors on the target tissues of the wasting process is adequately understood, the in vivo or in vitro effect on cancer cell and the tumor environment, in some cases, is almost entirely unknown. On this point, cancer cells may express the receptors for TGF $\beta$-like myokines and myostatin results in a pro-apoptotic effect when assayed in vitro on cancer cell lines (96), in addition myostatin inhibits tumor growth even in vivo $(19,96)$. Actually the restraint of neoplasia growth by a factor produced by the cancer cell itself is a somewhat unanticipated outcome. Moreover, the genetic or epigenetic alterations underlying expression of the pro-cachectic factor by cancer cells are unknown and even more so it is unknown whether these genomic changes may be regarded as driver or passenger mutations (97). The seemingly disagreeing results reported (64) in comparison to others $(53,63)$ may be evaluated according the intricacies of the overall pathophysiology of TGF $\beta$-like myokines.

In conclusion, the present study highlights the usefulness of introducing a measurement of tumor burden for a straightforward characterization of the cancer wasting process known as cachexia. In particular, the discussed approach may be a tool of discovery, even in humans, of new genetic polymorphisms affecting the host vulnerability to tumor growth and ensuing cachexia.

\section{Acknowledgements}

I would like to thank Elena Monti, Università Insubria for helpful discussion.

\section{References}

1. Tisdale MJ: Mechanisms of cancer cachexia. Physiol Rev 89: 381-410, 2009.

2. Fearon KC, Glass DJ and Guttridge DC: Cancer cachexia: Mediators, signaling, and metabolic pathways. Cell Metab 16: 153-166, 2012.

3. Argilés JM, Busquets S, Stemmler B and López-Soriano FJ Cancer cachexia: Understanding the molecular basis. Nat Rev Cancer 14: 754-762, 2014.

4. Ali S and Garcia JM: Sarcopenia, cachexia and aging: Diagnosis, mechanisms and therapeutic options - a mini-review. Gerontology 60: 294-305, 2014.

5. Fearon K, Strasser F, Anker SD, Bosaeus I, Bruera E, Fainsinger RL, Jatoi A, Loprinzi C, MacDonald N, Mantovani G, et al: Definition and classification of cancer cachexia: An international consensus. Lancet Oncol 12: 489-495, 2011.

6. Argilés JM, López-Soriano FJ, Toledo M, Betancourt A, Serpe R and Busquets S: The cachexia score (CASCO): A new tool for staging cachectic cancer patients. J Cachexia Sarcopenia Muscle 2: 87-93, 2011.

7. Windsor JA and Hill GL: Risk factors for postoperative pneumonia. The importance of protein depletion. Ann Surg 208 209-214, 1988.
8. Tian M, Nishijima Y, Asp ML, Stout MB, Reiser PJ and Belury MA: Cardiac alterations in cancer-induced cachexia in mice. Int J Oncol 37: 347-353, 2010.

9. Damrauer JS, Stadler ME, Acharyya S, Baldwin AS, Couch ME and Guttridge DC: Chemotherapy-induced muscle wasting: Association with NF- $\kappa \mathrm{B}$ and cancer cachexia. Basic Appl Myol 18: 139-148, 2008.

10. Sakai H, Sagara A, Arakawa K, Sugiyama R, Hirosaki A Takase K, Jo A, Sato K, Chiba Y, Yamazaki M, et al: Mechanisms of cisplatin-induced muscle atrophy. Toxicol Appl Pharmacol 278: 190-199, 2014.

11. Bachmann J, Heiligensetzer M, Krakowski-Roosen $\mathrm{H}$, Büchler MW, Friess H and Martignoni ME: Cachexia worsens prognosis in patients with resectable pancreatic cancer. J Gastrointest Surg 12: 1193-1201, 2008.

12. Onesti JK and Guttridge DC: Inflammation based regulation of cancer cachexia. Biomed Res Int 2014: 168407, 2014

13. Hotamisligil GS: The role of TNFalpha and TNF receptors in obesity and insulin resistance. J Intern Med 245: 621-625, 1999.

14. Heinrich PC, Behrmann I, Haan S, Hermanns HM, Müller-Newen G and Schaper F: Principles of interleukin (IL)-6-type cytokine signalling and its regulation. Biochem J 374: $1-20,2003$.

15. Schakman O, Kalista S, Barbé C, Loumaye A and Thissen JP: Glucocorticoid-induced skeletal muscle atrophy. Int J Biochem Cell Biol 45: 2163-2172, 2013.

16. Watchorn TM, Waddell I, Dowidar N and Ross JA: Proteolysisinducing factor regulates hepatic gene expression via the transcription factors NF-(kappa)B and STAT3. FASEB J 15: 562-564, 2001.

17. Deans DA, Wigmore SJ, Gilmour H, Tisdale MJ, Fearon KC and Ross JA: Expression of the proteolysis-inducing factor core peptide mRNA is upregulated in both tumour and adjacent normal tissue in gastro-oesophageal malignancy. Br J Cancer 94: 731-736, 2006.

18. Han HQ, Zhou X, Mitch WE and Goldberg AL: Myostatin/activin pathway antagonism: Molecular basis and therapeutic potential. Int J Biochem Cell Biol 45: 2333-2347, 2013.

19. Zimmers TA, Davies MV, Koniaris LG, Haynes P, Esquela AF, Tomkinson KN, McPherron AC, Wolfman NM and Lee SJ: Induction of cachexia in mice by systemically administered myostatin. Science 296: 1486-1488, 2002.

20. Elkina Y, von Haehling S, Anker SD and Springer J: The role of myostatin in muscle wasting: An overview. J Cachexia Sarcopenia Muscle 2: 143-151, 2011.

21. Martin L, Birdsell L, Macdonald N, Reiman T, Clandinin MT, McCargar LJ, Murphy R, Ghosh S, Sawyer MB and Baracos VE: Cancer cachexia in the age of obesity: Skeletal muscle depletion is a powerful prognostic factor, independent of body mass index. J Clin Oncol 31: 1539-1547, 2013.

22. Egerman MA and Glass DJ: Signaling pathways controlling skeletal muscle mass. Crit Rev Biochem Mol Biol 49: 59-68, 2014.

23. Nagy V and Dikic I: Ubiquitin ligase complexes: From substrate selectivity to conjugational specificity. Biol Chem 391: 163-169, 2010.

24. Baird TD and Wek RC: Eukaryotic initiation factor 2 phosphorylation and translational control in metabolism. Adv Nutr 3: 307-321, 2012.

25. Lecker SH, Jagoe RT, Gilbert A, Gomes M, Baracos V, Bailey J, Price SR, Mitch WE and Goldberg AL: Multiple types of skeletal muscle atrophy involve a common program of changes in gene expression. FASEB J 18: 39-51, 2004.

26. Petruzzelli M, Schweiger M, Schreiber R, Campos-Olivas R, Tsoli M, Allen J, Swarbrick M, Rose-John S, Rincon M, Robertson G, et al: A switch from white to brown fat increases energy expenditure in cancer-associated cachexia. Cell Metab 20: 433-447, 2014.

27. Bosaeus I, Daneryd P, Svanberg E and Lundholm K: Dietary intake and resting energy expenditure in relation to weight loss in unselected cancer patients. Int J Cancer 93: 380-383, 2001.

28. Tisdale MJ: Molecular pathways leading to cancer cachexia. Physiology (Bethesda) 20: 340-348, 2005

29. Hyltander A, Drott C, Körner U, Sandstrom R, Lundholm K: Elevated energy expenditure in cancer patients with solid tumours. Eur J Cancer 27: 9-15, 1991.

30. Cantor JR and Sabatini DM: Cancer cell metabolism: One hallmark, many faces. Cancer Discov 2: 881-898, 2012.

31. Wise DR and Thompson CB: Glutamine addiction: A new therapeutic target in cancer. Trends Biochem Sci 35: 427-433, 2010. 
32. Delano MJ and Moldawer LL: The origins of cachexia in acute and chronic inflammatory diseases. Nutr Clin Pract 21: 68-81, 2006.

33. Rousset S, Alves-Guerra MC, Mozo J, Miroux B, CassardDoulcier AM, Bouillaud F and Ricquier D: The biology of mitochondrial uncoupling proteins. Diabetes 53 (Suppl 1): S130-S135, 2004.

34. Bing C, Russell ST, BeckettEE, Collins P, Taylor S, Barraclough R, Tisdale MJ and Williams G: Expression of uncoupling proteins-1, -2 and -3 mRNA is induced by an adenocarcinoma-derived lipidmobilizing factor. Br J Cancer 86: 612-618, 2002.

35. Thair SA, Walley KR, Nakada TA, McConechy MK, Boyd JH, Wellman $\mathrm{H}$ and Russell JA: A single nucleotide polymorphism in $\mathrm{NF}-\kappa \mathrm{B}$ inducing kinase is associated with mortality in septic shock. J Immunol 186: 2321-2328, 2011

36. Tan BH, Ross JA, Kaasa S, Skorpen F and Fearon KC; European Palliative Care Research Collaborative: Identification of possible genetic polymorphisms involved in cancer cachexia: A systematic review. J Genet 90: 165-177, 2011.

37. Schneider DS, Ayres JS: Two ways to survive infection: What resistance and tolerance can teach us about treating infectious diseases. Nat Rev Immunol 8: 889-895, 2008

38. Råberg L, Graham AL and Read AF: Decomposing health: Tolerance and resistance to parasites in animals. Philos Trans R Soc Lond B Biol Sci 364: 37-49, 2009.

39. Li C, Corraliza I, Langhorne J: A defect in interleukin-10 leads to enhanced malarial disease in Plasmodium chabaudi chabaudi infection in mice. Infect Immun 67: 4435-4442, 1999

40. Medzhitov R, Schneider DS and Soares MP: Disease tolerance as a defense strategy. Science 335: 936-941, 2012.

41. De Lerma Barbaro A, Perletti G, Bonapace IM and Monti E: Inflammatory cues acting on the adult intestinal stem cells and the early onset of cancer (Review). Int J Oncol 45: 959-968, 2014

42. Wu ZH and Shi Y: When ubiquitin meets NF-кB: A trove for anticancer drug development. Curr Pharm Des 19: 3263-3275, 2013.

43. Karin M, Cao Y, Greten FR and Li ZW: NF-kappaB in cancer: From innocent bystander to major culprit. Nat Rev Cancer 2 : 301-310, 2002

44. Cai D, Frantz JD, Tawa NE Jr, Melendez PA, Oh BC, Lidoy HG, Hasselgren PO, Frontera WR, Lee J, Glass DJ, et al: IKKbeta/NF-kappaB activation causes severe muscle wasting in mice. Cell 119: 285-298, 2004

45. Hunter RB, Stevenson E, Koncarevic A, Mitchell-Felton H, Essig DA and Kandarian SC; SC: Activation of an alternative NF-kappaB pathway in skeletal muscle during disuse atrophy. FASEB J 16: 529-538, 2002.

46. Penner CG, Gang G, Wray C, Fischer JE and Hasselgren PO The transcription factors NF-kappab and AP-1 are differentially regulated in skeletal muscle during sepsis. Biochem Biophys Res Commun 281: 1331-1336, 2001.

47. Wyke SM, Russell ST, Tisdale MJ: Induction of proteasome expression in skeletal muscle is attenuated by inhibitors of NF-kappaB activation. Br J Cancer 91: 1742-1750, 2004

48. Wang H, Lai YJ, Chan YL, Li TL and Wu CJ: Epigallocatechin3 -gallate effectively attenuates skeletal muscle atrophy caused by cancer cachexia. Cancer Lett 305: 40-49, 2011.

49. Yang Q, Wan L, Zhou Z, Li Y, Yu Q, Liu L, Li B and Guo C: Parthenolide from Parthenium integrifolium reduces tumor burden and alleviate cachexia symptoms in the murine CT-26 model of colorectal carcinoma. Phytomedicine 20: 992-998, 2013.

50. Crawford LJ, Walker B and Irvine AE: Proteasome inhibitors in cancer therapy. J Cell Commun Signal 5: 101-110, 2011.

51. Beck SA, Smith KL and Tisdale MJ: Anticachectic and antitumor effect of eicosapentaenoic acid and its effect on protein turnover. Cancer Res 51: 6089-6093, 1991.

52. Whitehouse AS, Smith HJ, Drake JL and Tisdale MJ: Mechanism of attenuation of skeletal muscle protein catabolism in cancer cachexia by eicosapentaenoic acid. Cancer Res 61: 3604-3609, 2001.

53. Benny Klimek ME, Aydogdu T, Link MJ, Pons M, Koniaris LG and Zimmers TA: Acute inhibition of myostatin-family proteins preserves skeletal muscle in mouse models of cancer cachexia. Biochem Biophys Res Commun 391: 1548-1554, 2010.

54. Marchal JA, Lopez GJ, Peran M, Comino A, Delgado JR García-García JA, CondeV, Aranda FM, Rivas C, Esteban M et al: The impact of PKR activation: from neurodegeneration to cancer. FASEB J 28: 1965-1974, 2014

55. Eley HL and Tisdale MJ: Skeletal muscle atrophy, a link between depression of protein synthesis and increase in degradation. J Biol Chem 282: 7087-7097, 2007.
56. Eley HL, Russell ST and Tisdale MJ: Role of the dsRNA-dependent protein kinase (PKR) in the attenuation of protein loss from muscle by insulin and insulin-like growth factor-I (IGF-I). Mol Cell Biochem 313: 63-69, 2008

57. Eley HL, Russell ST and Tisdale MJ: Attenuation of muscle atrophy in a murine model of cachexia by inhibition of the dsRNAdependent protein kinase. Br J Cancer 96: 1216-1222, 2007.

58. Eley HL, Russell ST and Tisdale MJ: Effect of branched-chain amino acids on muscle atrophy in cancer cachexia. Biochem J 407: 113-120, 2007.

59. Davis TW, Zweifel BS, O'Neal JM, Heuvelman DM, Abegg AL, Hendrich TO and Masferrer JL: Inhibition of cyclooxygenase-2 by celecoxib reverses tumor-induced wasting. J Pharmacol Exp Ther 308: 929-934, 2004.

60. Hyde CA and Missailidis S: Inhibition of arachidonic acid metabolism and its implication on cell proliferation and tumour-angiogenesis. Int Immunopharmacol 9: 701-715, 2009.

61. Roth E: Immune and cell modulation by amino acids. Clin Nutr 26: 535-544, 2007

62. Ham DJ, Murphy KT, Chee A, Lynch GS and Koopman R: Glycine administration attenuates skeletal muscle wasting in a mouse model of cancer cachexia. Clin Nutr 33: 448-458, 2014.

63. Zhou X, Wang JL, Lu J, Song Y, Kwak KS, Jiao Q, Rosenfeld R, Chen Q, Boone T, Simonet WS, et al: Reversal of cancer cachexia and muscle wasting by ActRIIB antagonism leads to prolonged survival. Cell 142: 531-543, 2010.

64. Gallot YS, Durieux AC, Castells J, Desgeorges MM, Vernus B, Plantureux L, Rémond D, Jahnke VE, Lefai E, Dardevet D, et al: Myostatin gene inactivation prevents skeletal muscle wasting in cancer. Cancer Res 74: 7344-7356, 2014

65. Ruas JL, White JP, Rao RR, Kleiner S, Brannan KT, Harrison BC, Greene NP, Wu J, Estall JL, Irving BA et al: A PGC-1 $\alpha$ isoform induced by resistance training regulates skeletal muscle hypertrophy. Cell 151: 1319-1331, 2012

66. Braun TP, Grossberg AJ, Krasnow SM, Levasseur PR Szumowski M, Zhu XX, Maxson JE, Knoll JG, Barnes AP and Marks DL: Cancer- and endotoxin-induced cachexia require intact glucocorticoid signaling in skeletal muscle. FASEB J 27: 3572-3582, 2013

67. Kadmiel M and Cidlowski JA: Glucocorticoid receptor signaling in health and disease. Trends Pharmacol Sci 34: 518-530, 2013.

68. Schakman O, Kalista S, Barbé C, Loumaye A and Thissen JP: Glucocorticoid-induced skeletal muscle atrophy. Int J Biochem Cell Biol 45: 2163-2172, 2013

69. Das SK, Eder S, Schauer S, Diwoky C, Temmel H, Guertl B, Gorkiewicz G, Tamilarasan KP, Kumari P, Trauner M, et al: Adipose triglyceride lipase contributes to cancer-associated cachexia. Science 333: 233-238, 2011.

70. Kir S, White JP, Kleiner S, Kazak L, Cohen P, Baracos VE and Spiegelman BM: Tumour-derived PTH-related protein triggers adipose tissue browning and cancer cachexia. Nature 513: 100-104, 2014.

71. Hendifar A, Yang D, Lenz F, Lurje G, Pohl A, Lenz C, Ning Y, Zhang W and Lenz HJ: Gender disparities in metastatic colorectal cancer survival. Clin Cancer Res 15: 6391-6397, 2009.

72. Koo JH, Jalaludin B, Wong SK, Kneebone A, Connor SJ and Leong RW: Improved survival in young women with colorectal cancer. Am J Gastroenterol 103: 1488-1495, 2008.

73. al-Azzawi F and Wahab M: Estrogen and colon cancer: Current issues. Climacteric 5: 3-14, 2002.

74. Cosper PF and Leinwand LA: Cancer causes cardiac atrophy and autophagy in a sexually dimorphic manner. Cancer Res 71: 1710-1720, 2011.

75. Sandri M: Protein breakdown in muscle wasting: Role of autophagy-lysosome and ubiquitin-proteasome. Int J Biochem Cell Biol 45: 2121-2129, 2013.

76. Strassmann G, Masui Y, Chizzonite R and Fong M: Mechanisms of experimental cancer cachexia. Local involvement of IL-1 in colon-26 tumor. J Immunol 150: 2341-2345, 1993.

77. Piccinini AM and Midwood KS: DAMPening inflammation by modulating TLR signalling. Mediators Inflamm 2010: 672395, 2010.

78. Cannon TY, Guttridge D, Dahlman J, George JR, Lai V, Shores C, Buzková $\mathrm{P}$ and Couch ME: The effect of altered Toll-like receptor 4 signaling on cancer cachexia. Arch Otolaryngol Head Neck Surg 133: 1263-1269, 2007.

79. Vahle AK, Kerem A, Oztürk E, Bankfalvi A, Lang S and Brandau S: Optimization of an orthotopic murine model of head and neck squamous cell carcinoma in fully immunocompetent mice - role of toll-like-receptor 4 expressed on host cells. Cancer Lett 317: 199-206, 2012. 
80. Di Marco S, Cammas A, Lian XJ, Kovacs EN, Ma JF, Hall DT, Mazroui R, Richardson J, Pelletier J and Gallouzi IE: The translation inhibitor pateamine A prevents cachexia-induced muscle wasting in mice. Nat Commun 3: 896, 2012.

81. Kuznetsov G, Xu Q, Rudolph-Owen L, Tendyke K, Liu J, Towle M, Zhao N, Marsh J, Agoulnik S, Twine N, et al: Potent in vitro and in vivo anticancer activities of des-methyl, des-amino pateamine A, a synthetic analogue of marine natural product pateamine A. Mol Cancer Ther 8: 1250-1260, 2009.

82. Buck M and Chojkier M: Muscle wasting and dedifferentiation induced by oxidative stress in a murine model of cachexia is prevented by inhibitors of nitric oxide synthesis and antioxidants. EMBO J 15: 1753-1765, 1996.

83. Hall DT, Ma JF, Marco SD and Gallouzi IE: Inducible nitric oxide synthase (iNOS) in muscle wasting syndrome, sarcopenia, and cachexia. Aging (Albany NY) 3: 702-715, 2011.

84. Buchan JR and Parker R: Eukaryotic stress granules: The ins and outs of translation. Mol Cell 36: 932-941, 2009.

85. Pretto F, Ghilardi C, Moschetta M, Bassi A, Rovida A, Scarlato V, Talamini L, Fiordaliso F, Bisighini C, Damia G, et al: Sunitinib prevents cachexia and prolongs survival of mice bearing renal cancer by restraining STAT3 and MuRF-1 activation in muscle. Oncotarget 6: 3043-3054, 2015.

86. Bonetto A, Aydogdu T, Jin X, Zhang Z, Zhan R, Puzis L, Koniaris LG and Zimmers TA: JAK/STAT3 pathway inhibition blocks skeletal muscle wasting downstream of IL-6 and in experimental cancer cachexia. Am J Physiol Endocrinol Metab 303: E410-E421, 2012.

87. Gonzalez MC, Pastore CA, Orlandi SP and Heymsfield SB: Obesity paradox in cancer: New insights provided by body composition. Am J Clin Nutr 99: 999-1005, 2014.

88. Monitto CL, Berkowitz D, Lee KM, Pin S, Li D, Breslow M, O'Malley B and Schiller M: Differential gene expression in a murine model of cancer cachexia. Am J Physiol Endocrinol Metab 281: E289-E297, 2001.
89. Robert F, Mills JR, Agenor A, Wang D, DiMarco S, Cencic R, Tremblay ML, Gallouzi IE, Hekimi S, Wing SS, et al: Targeting protein synthesis in a Myc/mTOR-driven model of anorexiacachexia syndrome delays its onset and prolongs survival. Cancer Res 72: 747-756, 2012.

90. Cuenca AG, Cuenca AL, Winfield RD, Joiner DN, Gentile L, Delano MJ, Kelly-Scumpia KM, Scumpia PO, Matheny MK, Scarpace PJ, et al: Novel role for tumor-induced expansion of myeloid-derived cells in cancer cachexia. J Immunol 192: 6111-6119, 2014.

91. Baltgalvis KA, Berger FG, Pena MM, Davis JM, Muga SJ and Carson JA: Interleukin-6 and cachexia in $\mathrm{Apc}^{\mathrm{Min} /+}$ mice. Am J Physiol Regul Integr Comp Physiol 294: R393-R401, 2008

92. Velázquez KT, Enos RT, Narsale AA, Puppa MJ, Davis JM, Murphy EA and Carson JA: Quercetin supplementation attenuates the progression of cancer cachexia in $\mathrm{Apc}^{\mathrm{Min} /+}$ mice. $\mathrm{J}$ Nutr 144: 868-875, 2014.

93. Lyons SK: Advances in imaging mouse tumour models in vivo. J Pathol 205: 194-205, 2005.

94. Jain M, Nilsson R, Sharma S, Madhusudhan N, Kitami T, Souza AL, Kafri R, Kirschner MW, Clish CB and Mootha VK: Metabolite profiling identifies a key role for glycine in rapid cancer cell proliferation. Science 336: 1040-1044, 2012.

95. Rose ML, Madren J, Bunzendahl H and Thurman RG: Dietary glycine inhibits the growth of B16 melanoma tumors in mice. Carcinogenesis 20: 793-798, 1999.

96. Liu Y, Cheng H, Zhou Y, Zhu Y, Bian R, Chen Y, Li C, Ma Q, Zheng Q, Zhang Y, et al: Myostatin induces mitochondrial metabolic alteration and typical apoptosis in cancer cells. Cell Death Dis 4: e494, 2013.

97. Stratton MR, Campbell PJ and Futreal PA: The cancer genome. Nature 458: 719-724, 2009. 